
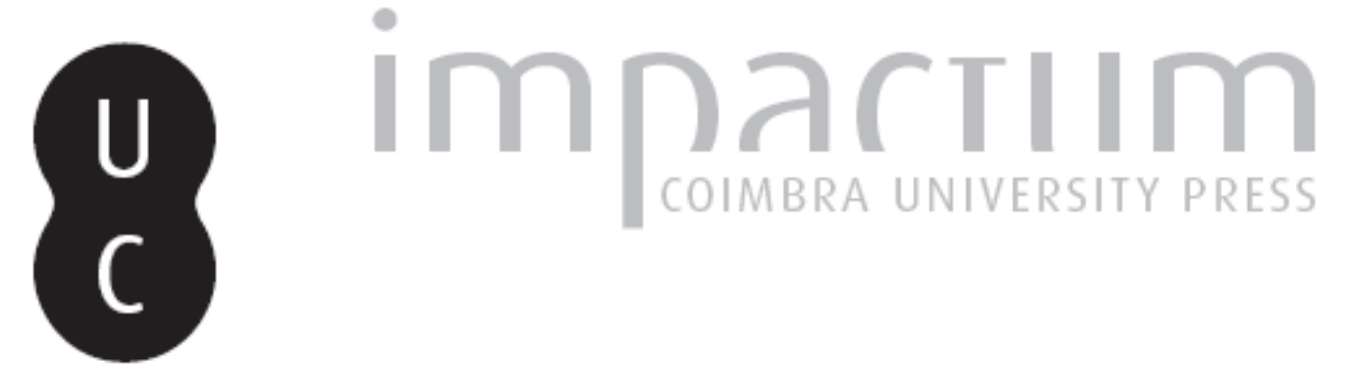

\title{
Mecenate: la "fortuna" di un nome
}

\section{Autor(es): $\quad$ Donati, Angela}
Publicado por: Faculdade de Letras da Universidade de Coimbra/Imprensa da Universidade de Coimbra

URL persistente:

URI:http://hdl.handle.net/10316.2/41868

DOI:

DOI:https://doi.org/10.14195/1647-8657_55_5

Accessed : $\quad$ 26-Apr-2023 14:33:31

A navegação consulta e descarregamento dos títulos inseridos nas Bibliotecas Digitais UC Digitalis, UC Pombalina e UC Impactum, pressupõem a aceitação plena e sem reservas dos Termos e Condições de Uso destas Bibliotecas Digitais, disponíveis em https://digitalis.uc.pt/pt-pt/termos.

Conforme exposto nos referidos Termos e Condições de Uso, o descarregamento de títulos de acesso restrito requer uma licença válida de autorização devendo o utilizador aceder ao(s) documento(s) a partir de um endereço de IP da instituição detentora da supramencionada licença.

Ao utilizador é apenas permitido o descarregamento para uso pessoal, pelo que o emprego do(s) título(s) descarregado(s) para outro fim, designadamente comercial, carece de autorização do respetivo autor ou editor da obra.

Na medida em que todas as obras da UC Digitalis se encontram protegidas pelo Código do Direito de Autor e Direitos Conexos e demais legislação aplicável, toda a cópia, parcial ou total, deste documento, nos casos em que é legalmente admitida, deverá conter ou fazer-se acompanhar por este aviso.

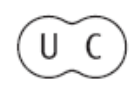


CONIMBRIGA

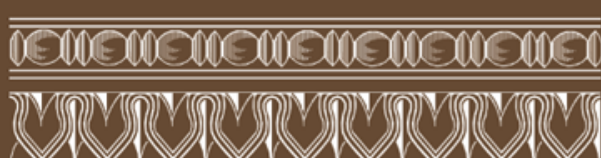

INSTITUTO DE ARQUEOLOGIA

VOLUME LV • 2016

FACULDADE DE LETRAS
UNIVERSIDADE DE COIMBRA 
Angela Donati

Università di Bologna

angela.donati@unibo.it

\section{MECENATE: LA “FORTUNA” DI UN NOME}

MAECENAS: THE "MEMORY" OF A NAME

“Conimbriga" LV (2016) p. 51-56

https://doi.org/10.14195/1647-8657_55_5

Riassunto: I diversi elementi che costituiscono il sistema onomastico di Mecenate si incontrano utilizzati per altri personaggi, tutti di origine libertina, e possono fornire indicazioni per ricostruire la familia dell'amico di Augusto.

Parole Chiave: Mecenate, onomastica.

Abstract: The components of the name-day system of Maecenas are used for other persons, all of libertine origin, and can provide elements to reconstruct the familia of the famous friend of Augustus.

KEYWORDS: Maecenas, name-day system. 
Página deixada propositadamente em branco 


\section{MECENATE: LA “FORTUNA” DI UN NOME}

Questa nota contiene alcune considerazioni di carattere onomastico e non fa in alcun modo riferimento al sostantivo derivato dal nome di Mecenate né al fenomeno del mecenatismo (ANDRÉ 1991: 105-159, con riferimento al capitolo Il mecenatismo: storia e leggenda) che ha costituito la "memoria" forse più persistente dell'aretino, almeno nell'immaginario colto, fino ai giorni nostri. L'agnomen Maecenatia$n u s$, derivato dall'onomastica di Mecenate, fu assunto precocemente, con l'intento preciso di evocare le azioni del più noto protettore delle arti, ma si perse ben presto: pochissime le attestazioni in questo senso, la più significativa delle quali appare in Marziale (Epigr., 8,55) ove il nome di Mecenate è espresso al plurale, nel significato attuale di mecenatismo (Sint Maecenates, non derunt Marones: se vi saranno dei mecenati, non mancheranno i poeti).

Ma l'esempio più famoso è offerto dal vanaglorioso Trimalcione; nell'opera di Petronio, infatti, dopo avere descritto il fastoso monumento funerario che desidera gli venga innalzato, rivolto ad Abinna, il ricco Trimalcione dà anche lettura dell'iscrizione che vuole vi sia incisa e che si apre con il suo nome: C. Pompeius Trimalchio Maecenatianus (Petr., Satyricon, 71, 12), in una forma nella quale l'agnomen tiene dietro ai tria nomina. E' ben nota la ricchezza nell'opera di Petronio di elementi onomastici (PriUli 1975) che richiamano momenti del passato greco e romano, soprattutto a sfondo mitico, espressi in forma ironica. Petronio/Trimalcione utilizza Maecenatianus come agnomen, ma la sua presenza nell'onomastica è nota anche in altri casi che possono forse collegarsi, anche se con motivazioni diverse, con il più illustre Mecenate (Solin 2001, che però non cita il caso di Mecenate).

Maecenas era utilizzato come cognomen da C. Cilnius e gli derivava, a quanto sembra, dalla famiglia paterna, della quale sono noti ad Arezzo alcuni componenti (FATUCCHI 1995: 187-205; BUONOCORE 
2009: 187-196)' ; questo elemento onomastico è portato da poche altre persone sicuramente diverse dal nostro. Maecenas è noto come gentilizio, soprattutto di liberti, e a Roma: sicuramente liberti di un C(aius) risultano essere C. Maecenas Elenchus (CIL VI, 7246, da un colombario), C. Maecenas Celer (CIL VI, 21773), C. Maecenas Eucarus e C. Maecenas Anteros (CIL VI, 21774), C. Maecenas Notus (CIL VI, 21776), C. Maecenas Capito (CIL VI, 35756), ai quali si aggiunge una donna, Maecenatia Hopora (CIL VI,21782); di altri appartenenti alla stessa gens non è esplicitata la posizione di liberti; fuori Roma vanno ricordati i casi di un aedilis menzionato in una iscrizione pubblica di Cartagine (AE 2011, 1703) e un secondo esempio da Igilgili (CIL VIII, 20213).

Anche il cognomen Maecenas/Mecenas è portato da poche altre persone, sicuramente diverse dal nostro e comunque, per diversi motivi, note; a queste si aggiunge il caso di una iscrizione proveniente da Roma (CIL VI, 23968): D. M. / C(aio) Pesidio Maxi/mo, v(ixit) a(nnis) $I V$. Fecit / L(ucius) Laelius Mece/nas filiaster. Il monumento non è conservato e l'iscrizione è pertanto nota solo dalla tradizione; nella forma tràdita essa pone, senza dubbio, qualche problema di interpretazione nel definire il rapporto fra le due persone menzionate nel testo che va probabilmente invertito riferendo l'ultima parola a Caio Pesidio Massimo e correggendola quindi in filiastro.

Va richiamata però l'attenzione sulla forma Maecenatianus/a, derivata da Maecenas, ed attestata in alcuni casi che possono suggerire un collegamento con l'aretino. Le testimonianze si riferiscono tutte a liberti imperiali e si raccolgono all'interno del sepolcreto dei servi e liberti di Livia, a Roma:

1: CIL VI, 4016: Cissus / Caesaris (servus) / Maecenat(ianus) // Parmeno / Liviae (servus) a purpur(a) / Maecenatian(o).

2: CIL VI, 4032: Agrypnus Caesar(is) / Aug(usti) (servus) Maecenatian(us) / a statuis.

3: CIL VI, 4095: Annia Liviae ... / Maecenatiana.

Da luoghi diversi di Roma provengono anche questi testi:

4: CIL VI, 19926 : C(aius) Iulius Aug(usti) l(ibertus) Delphus /

${ }^{1}$ Il volume 25 della Rivista Storica dell'Antichità (1995) raccoglie gli Atti di un Convegno tenuto ad Arezzo nel 1993, nel bimillenario della morte di Mecenate, con saggi di Riccardo Avallone, Mario Attilio Levi, Marta Sordi, Gabriella Poma, Giovannella Cresci, Remo Martini e Alberto Fatucchi.

Conimbriga, 55 (2016) 51-56 
Maecenatianus, / Iulia C(ai) l(iberta) Chronia, / Iulia C(ai) l(iberta) Secunda, / C(aius) Iulius Trophimus refecit sibi et suis / lib(ertis) libertabusque posterisque eor(um).

5: CIL VI, 22970: Buzyges / Augusti ser(vus) / Maecenat(ianus).

6: Notizie Scavi 1920, p. 36 (= AE 1921, 69): Apollonius / Neronis ser(vus) / Maecenatian[us].

Tutti questi servi e liberti imperiali si datano all'età di Augusto, compreso Apollonius, in quanto il Nerone del quale è servo è il figlio di Germanico e di Agrippina, non il futuro imperatore; alcuni hanno anche svolto funzioni non comuni, quale quelle dell'addetto a purpura o quello a statuis, mansioni che entrambe si collegano a persone di una certa cultura, particolarmente preparate ed affidabili nei rispettivi settori di competenza. Maecenatianus potrebbe non essere in questi casi un semplice agnomen, ma rivelare un collegamento con Mecenate, per analogia anche con l'aggettivo maecenatianus utilizzato da Plinio (Nat., 14,67), ma anche da altri autori che elencano i maecenatiana vina o i maecenatiani horti con preciso riferimento a quei vini che l'aretino produceva o a quei giardini che possedeva e che sono ben noti dalle fonti, anche per l'intensa attività culturale che ospitavano.

Tutti i beni di Mecenate passarono alla sua morte in eredità ad Augusto e anche il personale servile entrò a far parte del patrimonio dell'imperatore (WEAVER 2008: 212-213), compresa quella piccola corte di servi che aveva costituito la familia di Mecenate e che conservò nel nome il ricordo del precedente dominus.

\section{BIBLIOGRAFIA}

ANDRÉ Jean Marie (1991) - Mecenate. Un tentativo di biografia spirituale, Firenze (trad. it. dell'edizione Paris 1967).

Ben Abdallah Zeineb Benzina (2011) - Ladjimi Sebai Leila, Catalogue des inscriptions latine païennes inédites au Musée de Carthage, Rome, pp.83-84, n.114 (= AE 2011, 1703).

BuOnOCORE Marco (2009) - in Arezzo nell'Antichità, a cura di Giovanni Camporeale, Roma, pp. 187-196.

FATUCCHi Alberto (1995) - Le tracce della gens Cilnia nel territorio dell'Etruria, "Rivista Storica dell'Antichità", 25, pp. 187-205.

PriUli Stefano (1975) - Ascyltus. Note di onomastica petroniana, Bruxelle.

Solin Heikki (2001) - Un aspetto dell'onomastica plebea e municipale: la ripresa

Conimbriga, 55 (2016) 51-56 
di nomi illustri da parte di comuni cittadini, in Varia Epigraphica, Atti del Colloquio Internazionale di Epigrafia. Bertinoro, 8-10 giugno 2000, Faenza, pp. 411-427.

Weaver Paul R. C. (2008) - Familia Caesaris. A Study of the Emperor's Freedmen and Slaves, Cambridge M.

Conimbriga, 55 (2016) 51-56 